\title{
LEP II Beam Energy Measurement using Radiative Return Events
}

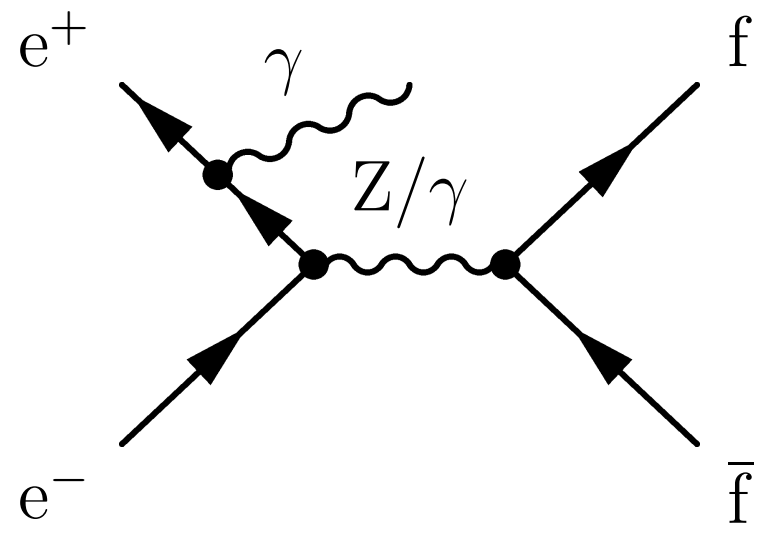

\section{Chris Ainsley}

University of Cambridge, UK

Presentation of preliminary results on behalf of the LEP Collaborations

\section{ICHEP2002 \\ Amsterdam}




\section{Overview}

- Motivation for a precise knowledge of the LEP beam energy $\left(E_{\mathrm{b}}\right)$.

- The radiative return approach to $E_{\mathrm{b}}$ (first reported on by ALEPH in Phys. Lett. B464 (1999) 339).

- Measurements by DELPHI, L3 and OPAL.

- Systematic studies.

- Results \& LEP combination.

- Conclusion. 


\section{Why determine $E_{\mathrm{b}}$ accurately?}

- Knowledge of $E_{\mathrm{b}}$ important for many precision measurements at LEP.

- Relevant for measurement of $\int \mathcal{L} d t$ via Bhabha cross-section $\Rightarrow$ fundamental to all cross-section determinations.

- Vital for accuracy of $m_{W}$ measurement; a principal objective of LEP II $\rightarrow$ resolution improved through kinematic fit constraints:

$$
\frac{\Delta m_{\mathrm{W}}}{m_{\mathrm{W}}}=\frac{\Delta E_{\mathrm{b}}}{E_{\mathrm{b}}} .
$$

- Standard LEP calibration relies on LEP I technique of resonant depolarisation at $E_{\mathrm{b}} \sim 40-60 \mathrm{GeV}$, together with magnetic extrapolation to LEP II energies $\left(E_{\mathrm{b}} \sim 100 \mathrm{GeV}\right)$, assuming $E_{\mathrm{b}}=a+b B$ $\Rightarrow$ indirect determination: need to be confident it really works at $\sim 20 \mathrm{MeV}$ level LEP claims. 


\section{Radiative Return Approach}

- $E_{\mathrm{b}}$ measured directly by LEP experiments.

- Use radiative fermion-pair events and construct

$\sqrt{s^{\prime}}=$ centre-of-mass energy after initial state radiation (ISR) .

$\mathrm{e}^{+}$
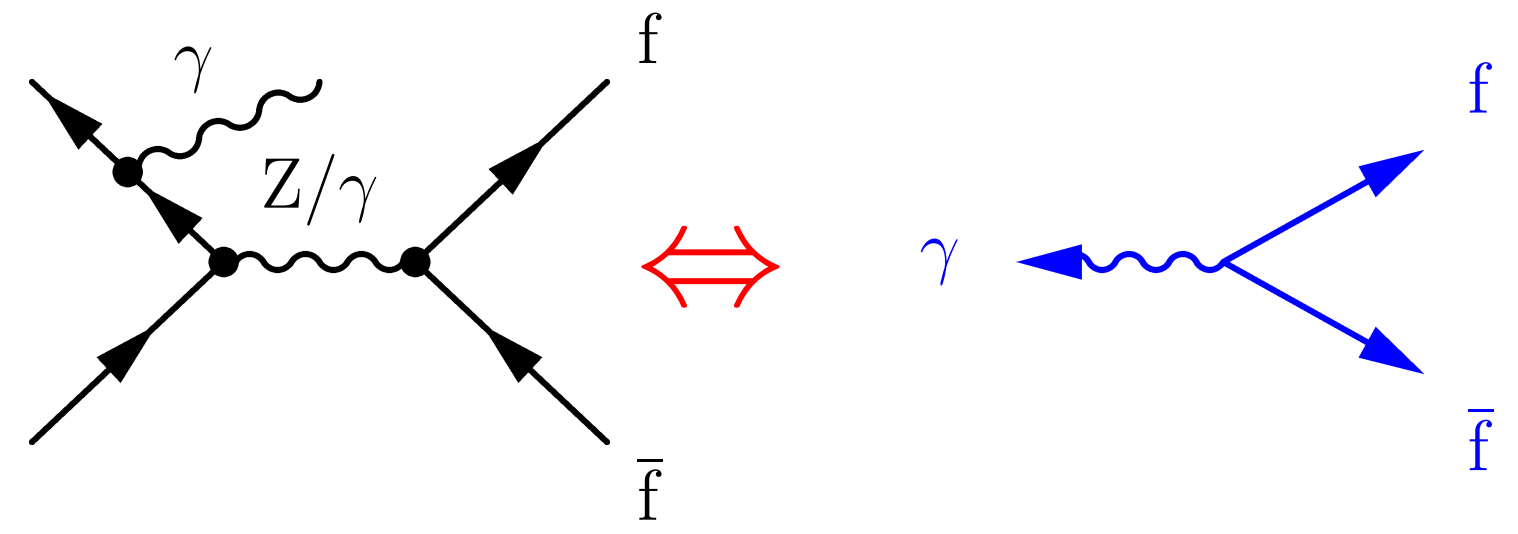

- $\sqrt{s^{\prime}}$ sensitive to $E^{\mathrm{LEP}}$ through energy and momentum constraints in kinematic fits.

- Use ISR events with $\sqrt{s^{\prime}} \sim m_{\mathrm{Z}}$ to reconstruct 'pseudo'-Z peak in MC $(\sqrt{s}$ known exactly $)$ and in data ( $\sqrt{s}$ known by measurement).

- Attribute any relative shift to a discrepancy in the measurement of the beam energy $\left(\Delta E_{\mathrm{b}}\right)$. 


\section{Reconstructed $\sqrt{s^{\prime}}$ Distribution}

- 1998-2000 L3 hadronic data $\left(627 \mathrm{pb}^{-1}\right)$ :

$E_{\mathrm{b}} \sim 94-104 \mathrm{GeV}$.

- $\sqrt{s^{\prime}}$ reconstructed from angles only:

$$
\sqrt{\frac{s^{\prime}}{s}}=\sqrt{\frac{\sin \chi_{\mathrm{q} \gamma}+\sin \chi_{\overline{\mathrm{q}} \gamma}-\left|\sin \left(\chi_{\mathrm{q} \gamma}+\chi_{\overline{\mathrm{q}} \gamma}\right)\right|}{\sin \chi_{\mathrm{q} \gamma}+\sin \chi_{\overline{\mathrm{q}} \gamma}+\left|\sin \left(\chi_{\mathrm{q} \gamma}+\chi_{\overline{\mathrm{q}} \gamma}\right)\right|}} .
$$

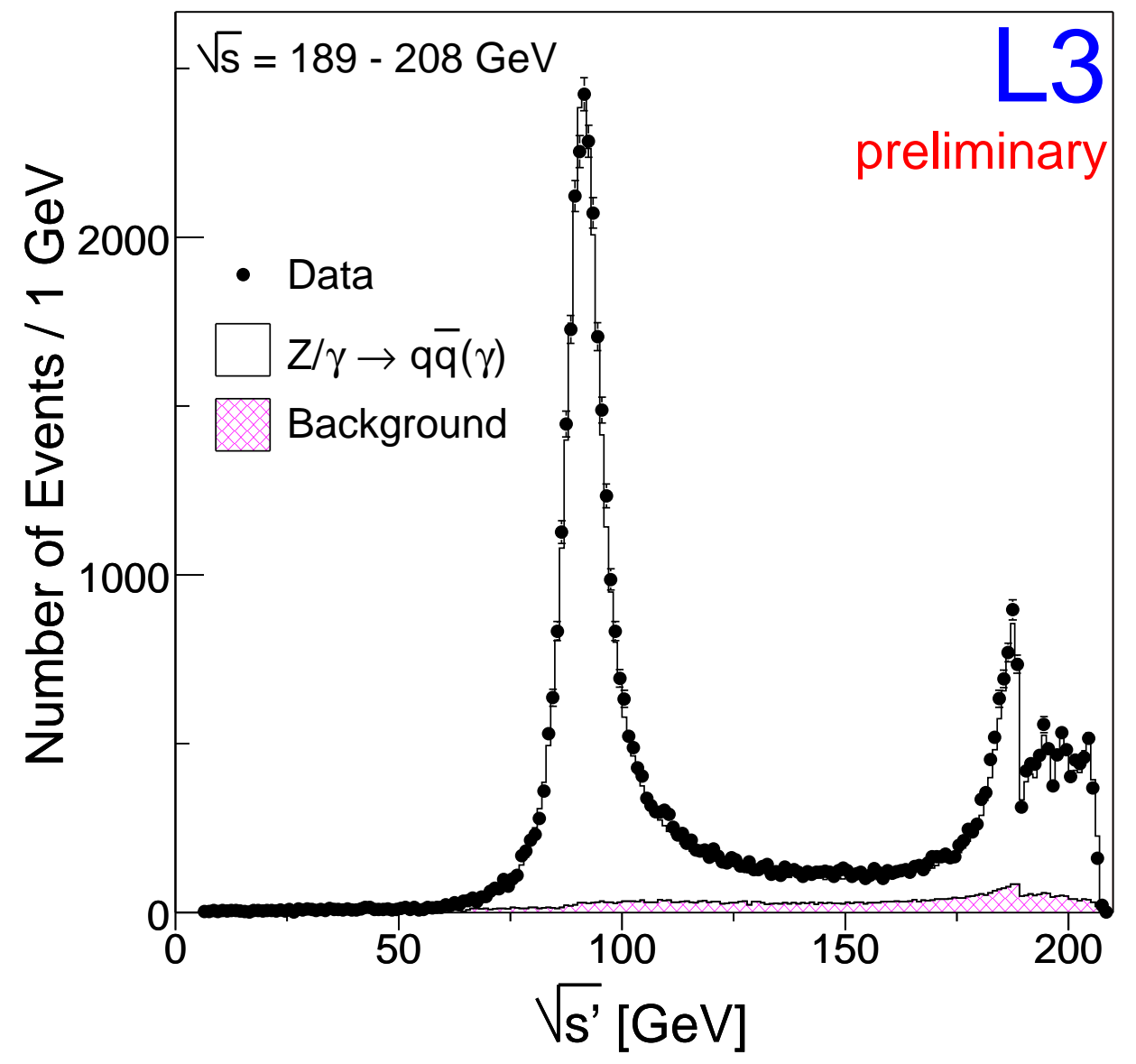

- Dominated by events under radiative return peak ( $\approx$ independent of $E_{\mathrm{b}}$ ) and at full energy. 


\section{$\Delta E_{\mathrm{b}}$ from $\mathrm{e}^{+} \mathrm{e}^{-} \rightarrow \mathrm{q} \overline{\mathrm{q}} \gamma$}

(1) Re-weighting method (DELPHI, L3):

- Use knowledge of $m_{\mathrm{Z}}$ from LEP I.

- Re-weight Monte Carlo $\sqrt{s^{\prime}}$ distribution with Breit-Wigner-like function $f\left(s^{\prime}, m_{\mathrm{Z}}\right)$ assuming different $m_{\mathrm{Z}}^{\text {new }}$ :

$$
w\left(s^{\prime}, m_{\mathrm{Z}}^{\text {new }}\right)=\frac{f\left(s^{\prime}, m_{\mathrm{Z}}^{\text {new }}\right)}{f\left(s^{\prime}, m_{\mathrm{Z}}^{\mathrm{LEP}}\right)} .
$$

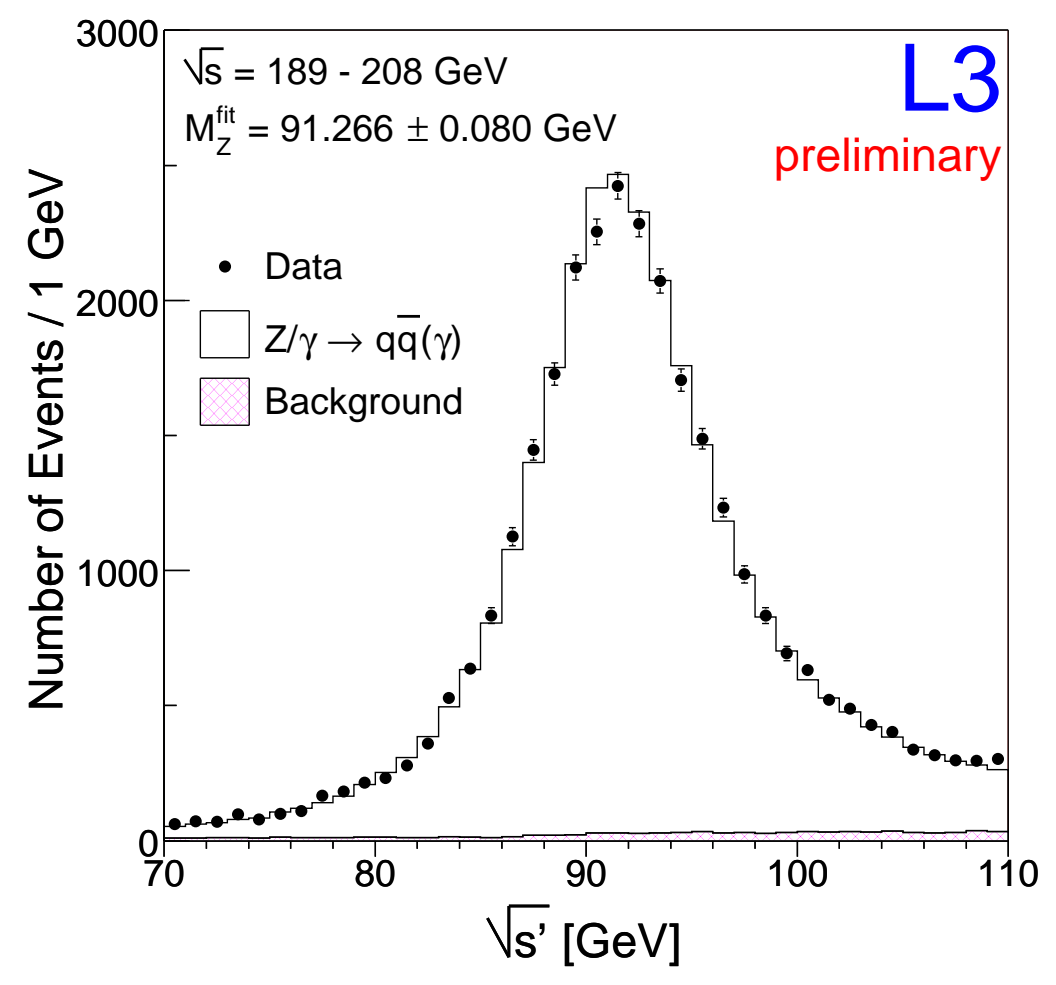

- Optimise agreement with data by varying $m_{\mathrm{Z}}^{\text {new }}$

$$
\Rightarrow \Delta E_{\mathrm{b}}=-E_{\mathrm{b}}\left(\frac{m_{\mathrm{Z}}^{\text {new }}-m_{\mathrm{Z}}^{\mathrm{LEP}}}{m_{\mathrm{Z}}^{\mathrm{LEP}}}\right) .
$$




\section{$\Delta E_{\mathrm{b}}$ from $\mathrm{e}^{+} \mathrm{e}^{-} \rightarrow \mathrm{q} \overline{\mathrm{q}} \gamma$}

(2) Fit method (OPAL):

- Fit Breit-Wigner-like function to $\sqrt{s^{\prime}}$ distribution in MC (at known $\sqrt{s}$ ) around 'pseudo'-Z peak.

- Fit same function to $\sqrt{s^{\prime}}$ distribution in data as a function of $\Delta E_{\mathrm{b}}=E_{\mathrm{b}}^{\mathrm{OPAL}}-E_{\mathrm{b}}^{\mathrm{LEP}}$, allowing normalisation/peak position $\left(M^{*}\right)$ to vary.
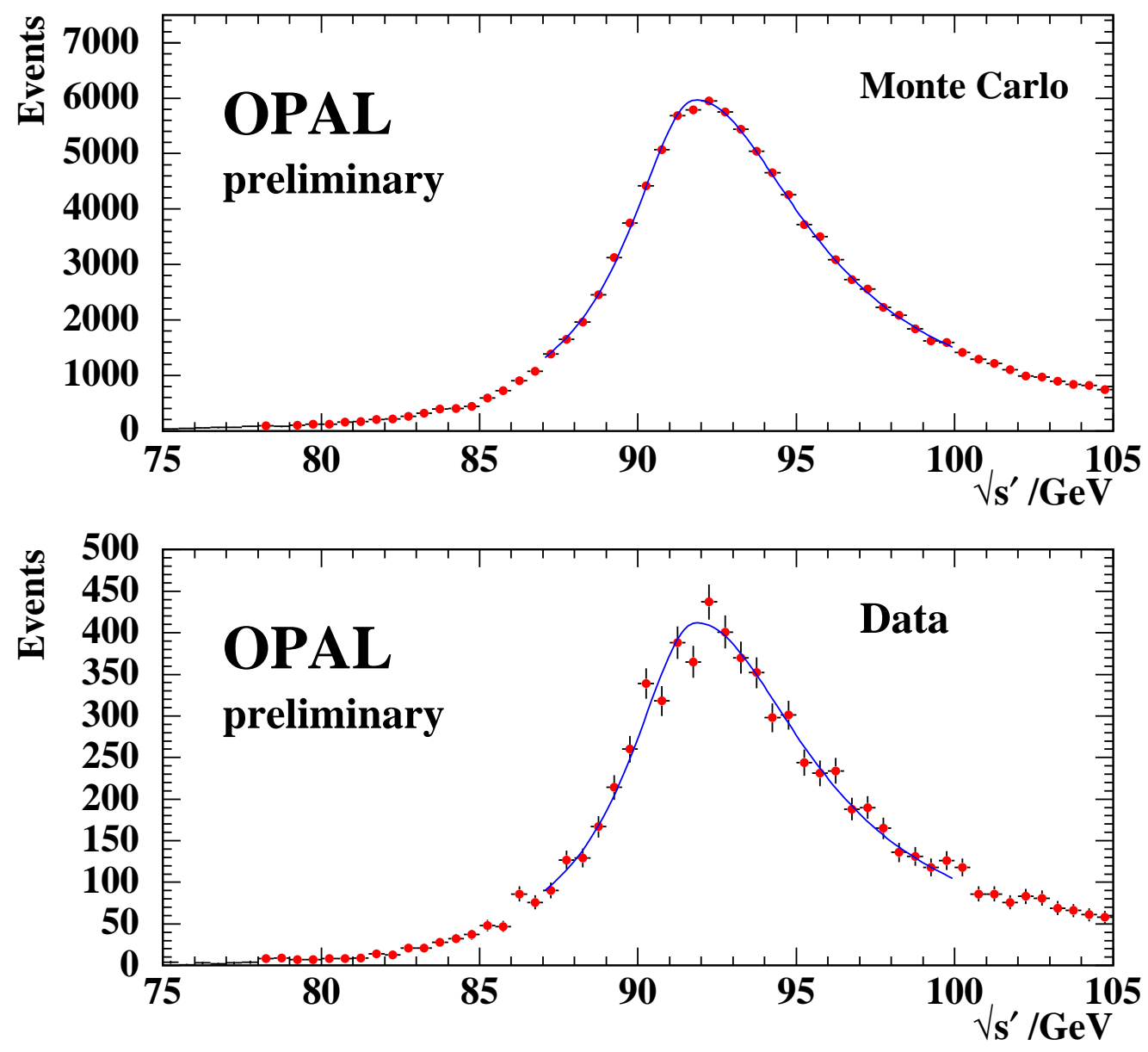

- Extract $\Delta E_{\mathrm{b}}$ from $M_{\text {data }}^{*}\left(\Delta E_{\mathrm{b}}\right)=M_{\mathrm{MC}}^{*}$. 


\section{$\Delta E_{\mathrm{b}}$ from $\mathrm{e}^{+} \mathrm{e}^{-} \rightarrow \ell^{+} \ell^{-} \gamma$}

Fit method (DELPHI: $\ell=\mu$; OPAL: $\ell=\mathrm{e}, \mu, \tau$ ):

- Define $x \equiv \sqrt{\frac{s^{\prime}}{s}}$.

- Construct $\Delta E_{\mathrm{cms}}=\frac{m_{\mathrm{Z}}}{x}-\sqrt{s}$ for each event.

- Fit Breit-Wigner-like function to distribution of $\Delta E_{\mathrm{cms}}$ in Monte Carlo and in data:

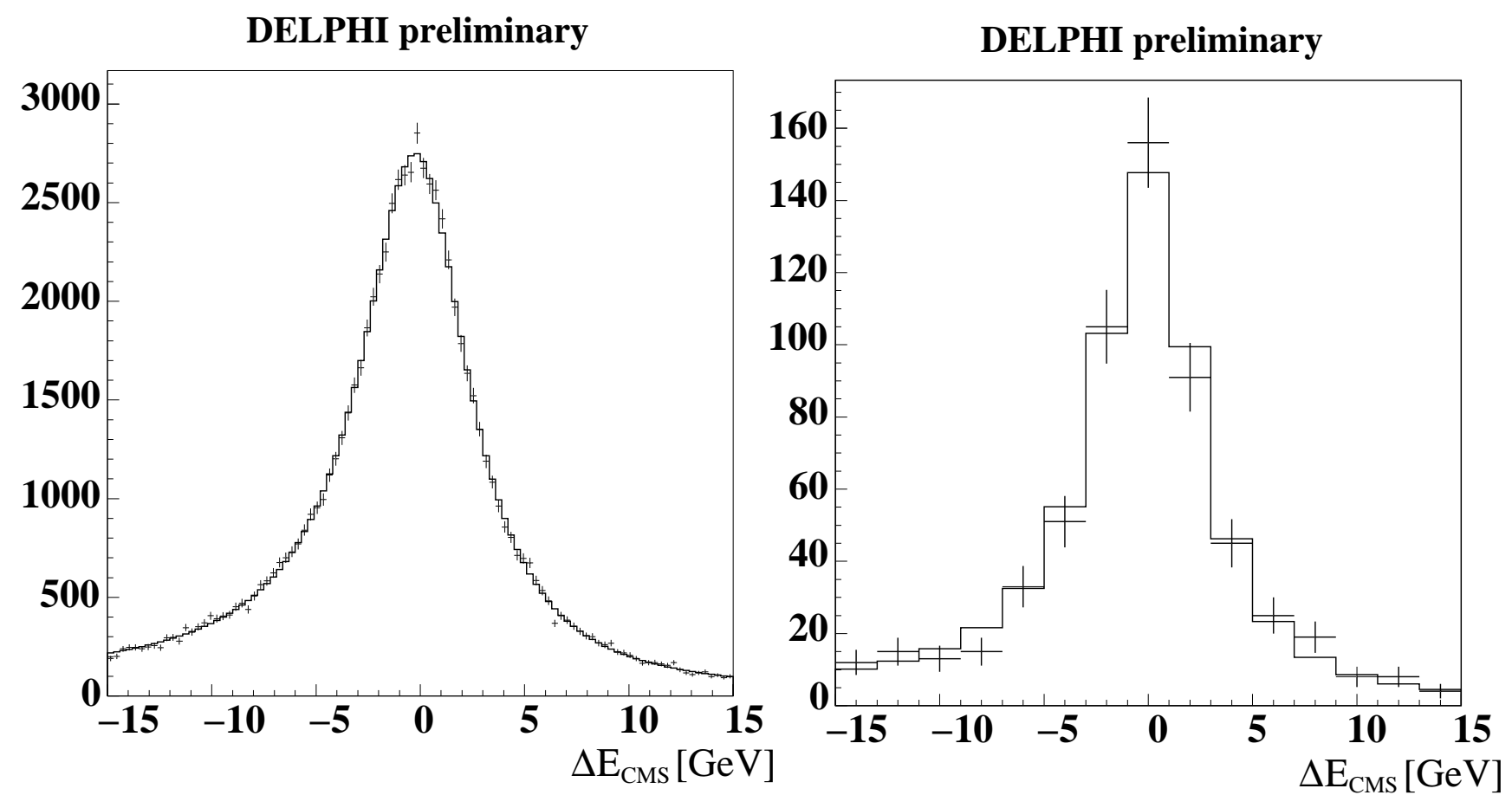

- Extract relative difference in peaks:

$$
\Rightarrow \Delta E_{\mathrm{b}}=\frac{1}{2}\left(\Delta E_{\mathrm{cms}}^{\mathrm{data}}-\Delta E_{\mathrm{cms}}^{\mathrm{MC}}\right) .
$$




\section{Systematic Studies}

- Motivated by $m_{\mathrm{W}}$ systematics; dominated by detector modelling and quark fragmentation.

\begin{tabular}{|c|c|c|c|}
\hline Experiment & $\begin{array}{l}\text { Dominant systematics } \\
\qquad / \mathrm{MeV}\end{array}$ & & $\begin{array}{l}\text { Total } \\
/ \mathrm{MeV}\end{array}$ \\
\hline 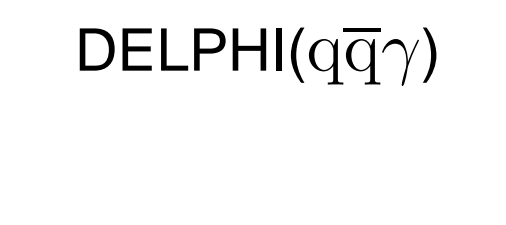 & $\begin{array}{l}\text { Data/MC tracking mismatch } \\
\text { Angular scale }\end{array}$ & $\begin{array}{l}60 \\
20\end{array}$ & 65 \\
\hline $\mathrm{L} 3(\mathrm{q} \overline{\mathrm{q}} \gamma)$ & $\begin{array}{l}\text { Jet energies } \\
\text { Angular scale }\end{array}$ & $\begin{array}{l}46 \\
40\end{array}$ & 75 \\
\hline OPAL $(q \bar{q} \gamma)$ & $\begin{array}{l}\text { Fragmentation } \\
\text { Jet/photon energies/angles }\end{array}$ & $\begin{array}{l}61 \\
29\end{array}$ & 70 \\
\hline $\operatorname{DELPHI}\left(\mu^{+} \mu^{-} \gamma\right)$ & Angular scale & 24 & 27 \\
\hline $\operatorname{OPAL}\left(\mathrm{e}^{+} \mathrm{e}^{-} \gamma\right)$ & $\begin{array}{l}\text { MC stats } \\
\text { Angular scale }\end{array}$ & $\begin{array}{l}52 \\
43\end{array}$ & 78 \\
\hline $\operatorname{OPAL}\left(\mu^{+} \mu^{-} \gamma\right)$ & Angular scale & 20 & 22 \\
\hline $\operatorname{OPAL}\left(\tau^{+} \tau^{-} \gamma\right)$ & Angular scale & 140 & 148 \\
\hline
\end{tabular}

- ISR modelling in $\mathcal{K} \mathcal{K} 2 f \mathrm{MC}$, backgrounds, fit parameters give only small uncertainties. 


\section{Results from DELPHI}

- 1997-2000 q $\overline{\mathrm{q}} \gamma$ data:

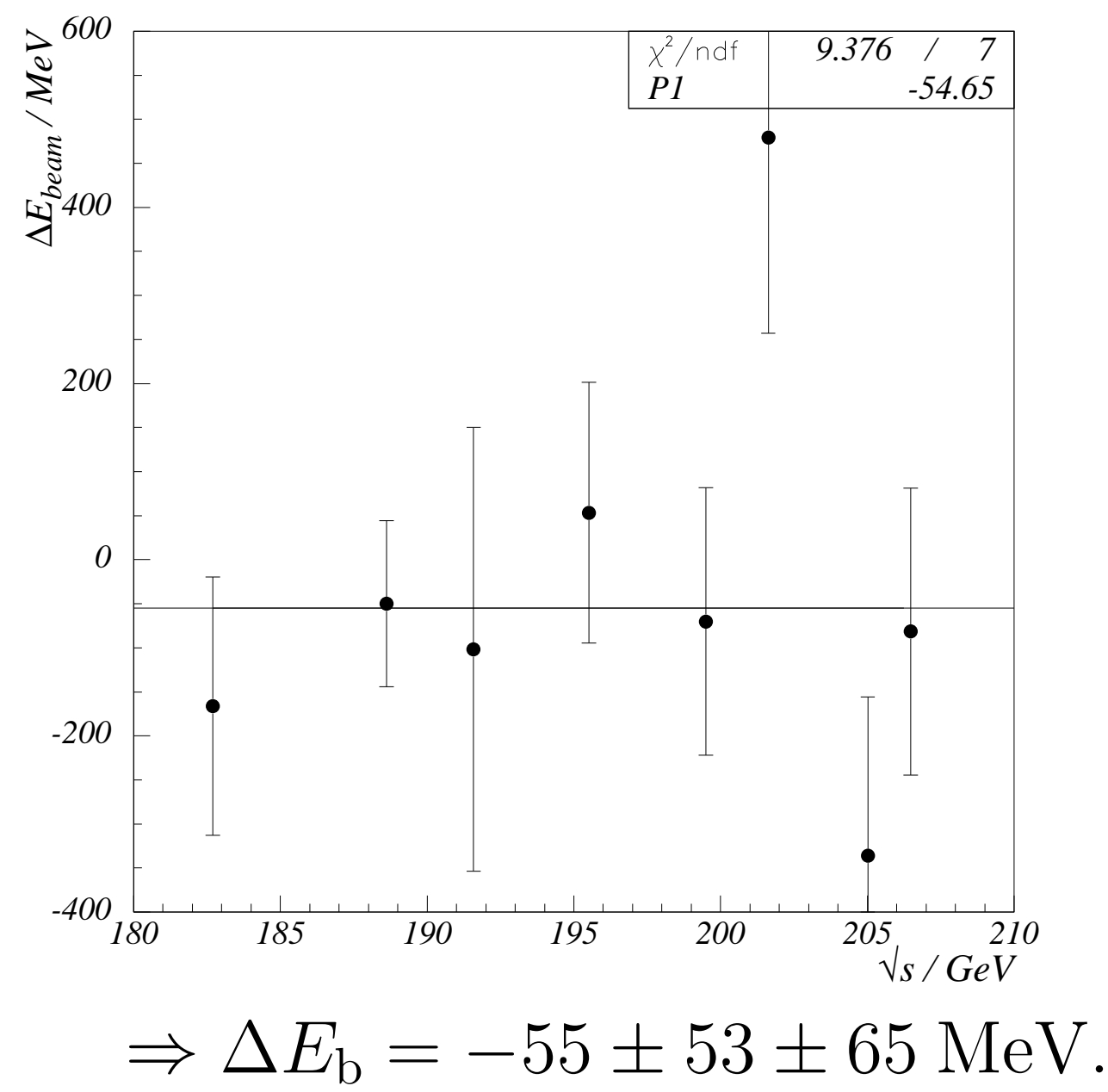

- 1997-2000 $\mu^{+} \mu^{-} \gamma$ data:

$\Rightarrow \Delta E_{\mathrm{b}}=+113 \pm 75 \pm 27 \mathrm{MeV}$.

- DELPHI combined:

$\Rightarrow \Delta E_{\mathrm{b}}=+34 \pm 47 \pm 37 \mathrm{MeV}$. 


\section{Results from L3}

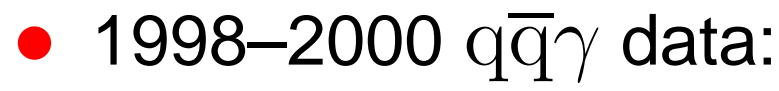

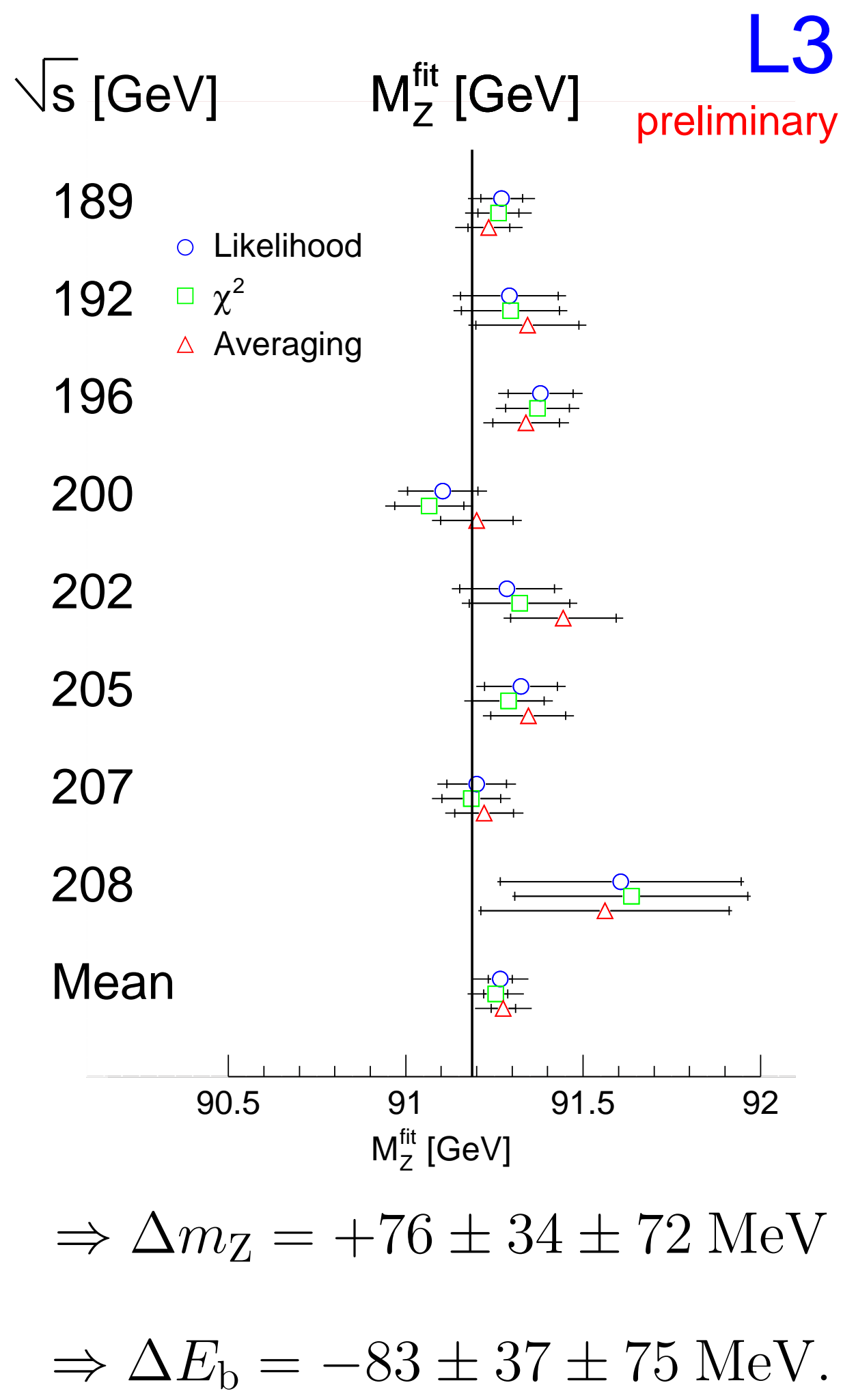




\section{Results from OPAL}

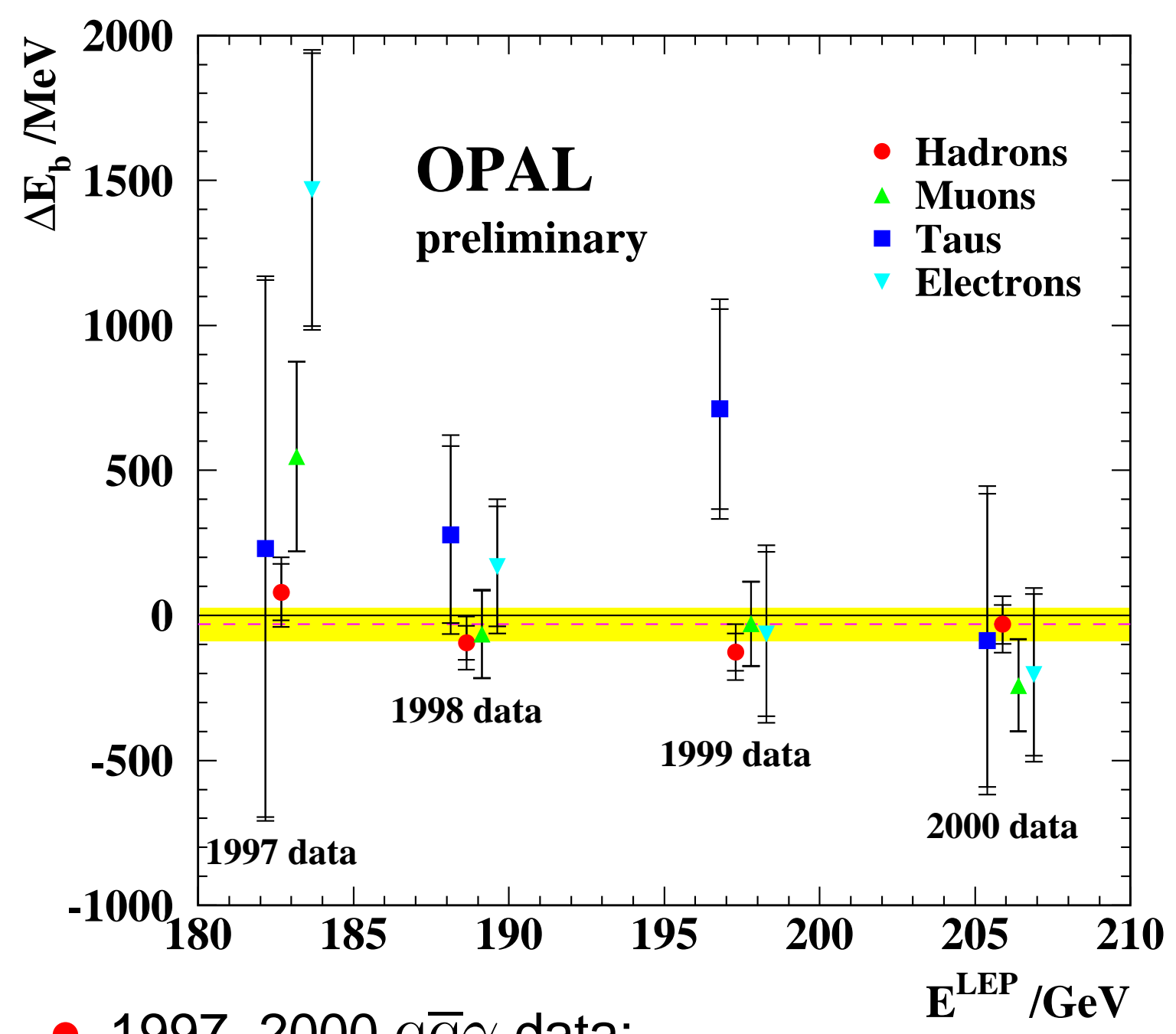

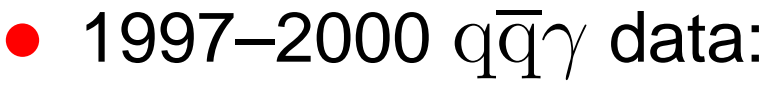

$$
\Rightarrow \Delta E_{\mathrm{b}}=-65 \pm 34 \pm 70 \mathrm{MeV} \text {. }
$$

- 1997-2000 $\ell^{+} \ell^{-} \gamma(\ell=\mathrm{e}, \mu, \tau)$ data:

$$
\Rightarrow \Delta E_{\mathrm{b}}=-1 \pm 68 \pm 26 \mathrm{MeV} \text {. }
$$

- OPAL combined:

$$
\Rightarrow \Delta E_{\mathrm{b}}=-31 \pm 40 \pm 36 \mathrm{MeV} .
$$




\section{Combination}

- Summary of all radiative return results:

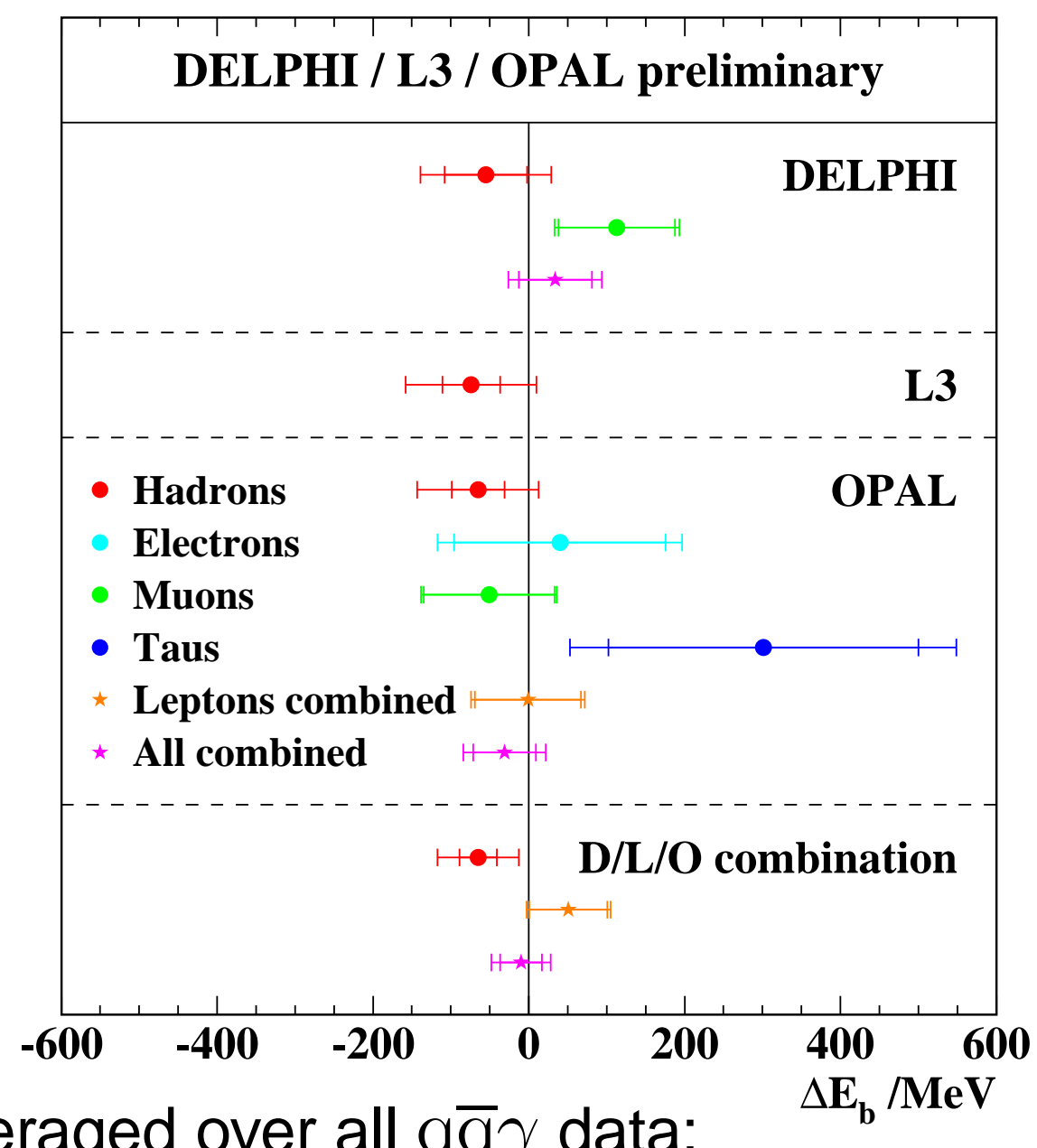

- Averaged over all $q \bar{q} \gamma$ data: $\quad \Delta \mathbf{E}_{\mathbf{b}} / \mathbf{M e V}$

$$
\Rightarrow \Delta E_{\mathrm{b}}=-65 \pm 24 \pm 45 \mathrm{MeV} \text {. }
$$

- Averaged over all $\ell^{+} \ell^{-} \gamma$ data:

$$
\Rightarrow \Delta E_{\mathrm{b}}=+51 \pm 50 \pm 19 \mathrm{MeV} \text {. }
$$

- Averaged over all data:

$$
\Rightarrow \Delta E_{\mathrm{b}}=-10 \pm 27 \pm 26 \mathrm{MeV} \text {. }
$$




\section{Conclusions}

- Beam energy from radiative returns is entirely consistent with standard LEP calibration $\Rightarrow$ good news for LEP calibration team; $\Rightarrow$ good news for $m_{\mathrm{W}}$ determination.

- Radiative return systematics $\sim$ uncertainty on magnetic extrapolation.

- Approach works with/without circulating beams $\Rightarrow$ potential method for evaluating $E_{\mathrm{b}}$ at a high-statistics future linear collider. 